\title{
The use of process simulation models in virtual commissioning of process automation software in drinking water treatment plants
}

\author{
G.I.M. Worm ${ }^{1 / 2}$, J.P. Kelderman ${ }^{1 / 3}$, T. Lapikas ${ }^{4}$, A.W.C. van der Helm ${ }^{5 / 2}$, K.M. van Schagen ${ }^{6}$, L.C. Rietveld ${ }^{2}$ \\ ${ }^{1}$ PWN, PO Box 2113, 1990 AC, Velserbroek, the Netherlands (e-mail: ignaz.worm@pwn.nl) \\ ${ }^{2}$ Delft University of Technology, Faculty of Civil Engineering and Geosciences, Department of Water \\ Management, PO Box 5048, 2600 GA, Delft, the Netherlands (e-mail: l.c.rietveld@tudelft.nl) \\ ${ }^{3}$ Nspyre, Poortweg 10, 2612 PA, Delft, the Netherlands (e-mail: jean-paul.kelderman@nspyre.nl) \\ ${ }^{4}$ UReason, Pompoenweg 9, 2321 DK, Leiden, the Netherlands (e-mail: tlapikas@ureason.com) \\ ${ }^{5}$ Waternet, PO Box 94370, 1090 GJ, Amsterdam, the Netherlands \\ (e-mail: alex.van.der.helm@waternet.nl) \\ ${ }^{6}$ Royal HaskoningDHV, PO Box 1132, 3800 BC, Amersfoort, the Netherlands \\ (e-mail:kim.van.schagen@rhdhv.com)
}

\begin{abstract}
This research deals with the contribution of process simulation models to the factory acceptance test (FAT) of process automation (PA) software of drinking water treatment plants. Two test teams tested the same piece of modified PA-software. One team used an advanced virtual commissioning (AVC) system existing of PAemulation and integrated process simulation models, the other team used the same PA-emulation but basic parameter relations instead of the process simulation models, the VC-system. Each test team found one (different) error of the thirteen errors put into the software prior to the experiment; the majority of the errors was found prior to the functional test. The team using the AVC-system found three errors, the team using the VC-system found four, but the AVC-team judged 1\% of the test items 'not possible', the VC-team 17\%. It was concluded that the hypothesis that with AVC more errors could be found than with VC could not be accepted. So, for the FAT of PAsoftware of drinking water treatment plants, the addition of basic parameter relations to PA-emulation satisfied. Not the exact process behavior helped to find errors, but the passing of process thresholds.
\end{abstract}

\section{Keywords}

Virtual commissioning; drinking water treatment; process automation; emulation; process simulation model

\section{INTRODUCTION}

As in other industries the level of automation of drinking water treatment plants has increased up to the level of remote multi-task supervisory control (Sheridan, 2002). New process automation (PA) software or software updates are tested extensively to prevent dangerous situations, process disturbances and down-time during or after implementation. At drinking water company PWN, in 2010, $1.7 \%$ of the urgent alarms occurred as a consequence of a PA-software modifications. Software testing is expensive, since it requires significant efforts of software experts. Still, to correct an error after implementation of the software, costs five to hundred times more (Poon et al., 2011).

The testing of PA-software can be divided in two main phases, i) the factory acceptance test (FAT) by the supplier in the development environment and ii) the site acceptance test (SAT) by the user in the live environment (Lucas, 2003), see Table 1. In this research, as is common at PWN, the system test and customer acceptance test (CAT) -both components of the FAT- are combined. This combined test is called the functional test. Notwithstanding the leading role of the supplier in the FAT, the user is involved in the CAT or functional test. Note that often in practice the CAT or functional test are referred to as FAT. 
Table 1. PA-software testing, based on (Lucas, 2003). FAT is factory acceptance test, SAT is site acceptance test.

\begin{tabular}{llll}
\hline Phase & Leading role & Location & Sub phase \\
\hline FAT & Supplier & Development environment & Unit test \\
& & & System test \\
& & Customer acceptance test \\
SAT & \multirow{2}{*}{ User } & Live environment & $\begin{array}{l}\text { Installation qualification } \\
\text { Operational qualification }\end{array}$ \\
& & & User acceptance test \\
\hline
\end{tabular}

To start a traditional FAT, the new or modified software is uploaded from the engineering station to a physical test-PLC (programmable logic controller) in an offline environment. The input/output (I/O) signals are simulated with physical switches or with a tailor made tool, for example programmed in Visual Basic or within the tested process automation software itself. To test the communication between PLCs and functions with interactions between multiple PLCs, a network must be set up between the PLCs. A single human-machine interface (HMI) client is available for navigation. In the traditional FAT, often new PA-software is extended with code exclusively for reasons of simulation during testing. These code lines are removed or disabled after the FAT, before the tested software is uploaded to the PLC in the plant.

A new development in the process automation software engineering is virtual commissioning (VC) instead of the traditional FAT. VC is the testing of software in a near-reality situation, using multiple virtual PLCs, or soft-controllers, containing emulated PA-software, their mutual communication, multiple HMI-clients possibly covering different hierarchical automation levels, virtual I/O and, possibly, basic parameter relations. The virtual I/O can be seen as equipment simulation and can be standardized by using typicals. The virtual I/O signals can be dynamical since the signals can be ramped or delayed. Examples of basic parameter relations are the relation between a pump's speed and the passing flow, or between the net flow to or from a tank and its level. A relevant characteristic of the modern emulation platforms like Siemens' Simit (Töbermann \& Fischer, 2007) and ABB's 800xA Simulator (Franke \& Doppelhamer, 2007) is the possibility to transfer the PA-software from the field PLC to the soft-controller without changing the software at all, and vice versa. This saves time and limits the risk of errors as a consequence of (not) changing the software before the transfer.

During VC of the control of the Hammerfest LNG plant in Norway more than 500 items were detected and resolved (Krause, 2007). VC has a saving potential of 10-30\% in the areas of test and commissioning of PA-software, in addition to the positive effects of VC on reductions of commissioning times and increase of quality of engineering solutions (Drath et al., 2008). In the automotive industry time savings in software development are estimated of more than $20 \%$ for commissioning using VC (Pellicciari et al., 2009; Wildemann, 2005). Within the software development process VC leads to overall economic savings of 20-50\% (Reinhart \& Wünsch, 2007). A field study has shown the added value of $\mathrm{VC}$ to software testing in a simulated production environment. Thirty persons using VC managed to fulfill an average of $85 \%$ of the requirements, as where a reference group realized $37 \%$. The $\mathrm{VC}$ testers needed $25 \%$ of the commissioning time of the 'traditional commissioning'-group (Zäh et al., 2006).

Two types of I/O can be distinguished, 'process I/O', signals of online measurements, commands and setpoints for pumps and valves, and 'status I/O', signals like equipment or system statuses. By connecting process simulation models to the 'process $\mathrm{I} / \mathrm{O}$ ' of the $\mathrm{VC}$-system dynamic process behavior is added. Advanced VC (AVC) is defined as VC with added process simulation models. As can be expected, the setup of an AVC-system existing of an emulated PA-system and process simulation, shows great similarities with the setup of a PA-system which is connected to the sensors 
and actuators in the field (Bradu et al., 2009). The objective of this research is to limit the process disturbances and downtime of drinking water treatment plants during and after the implementation of new PA-software or software updates. The hypothesis is that with AVC more errors will be found during the FAT than with VC.

\section{MATERIALS AND METHODS}

Test systems

The first application of VC with Simit in drinking water treatment by water supply company PWN in 2011 was the test of the update of the mutual communication of PLCs of the membrane filtration plant Heemskerk I. Errors which were in the existing software for more than ten years were found and solved. The first three projects tested with Simit within PWN yielded SATs without unexpected errors or delays, which was reason to double the number of licenses of the VC-platform. Programmers experienced that the border between software development and testing is vanishing. Particularly, they appreciated the possibilities to transfer software between the emulation platform and the PLC without needing to change the software and to emulate multiple PLCs and their mutual communication. AVC was not applied in drinking water treatment plants prior to this research.

Two test systems were used. The AVC-system existed of an emulation of the PA-software, of I/O virtualization and a process simulator, see Figure 1 (left). For emulation and virtualization of I/O, Simit was used. As is common for the PA-system, for visualization of the HMI, WinCC was used. In this research the embedded Object linking and embedding for Process Control (OPC) communication protocol was used to communicate with the process simulator Waterspot (Worm et al., 2010) running on the $\mathrm{USE}^{\circledR}$ platform. The process simulator processed 93 'process $\mathrm{I} / \mathrm{O}^{\prime}$ parameters dynamically, in real-time. Waterspot hosts two process simulation models. The hydraulic model (Worm et al., 2009) is set up in EPANET (Rossman, 2000), the water quality model (Worm et al., under review) is a Stimela model (Van der Helm \& Rietveld, 2002; Van Schagen, 2009).

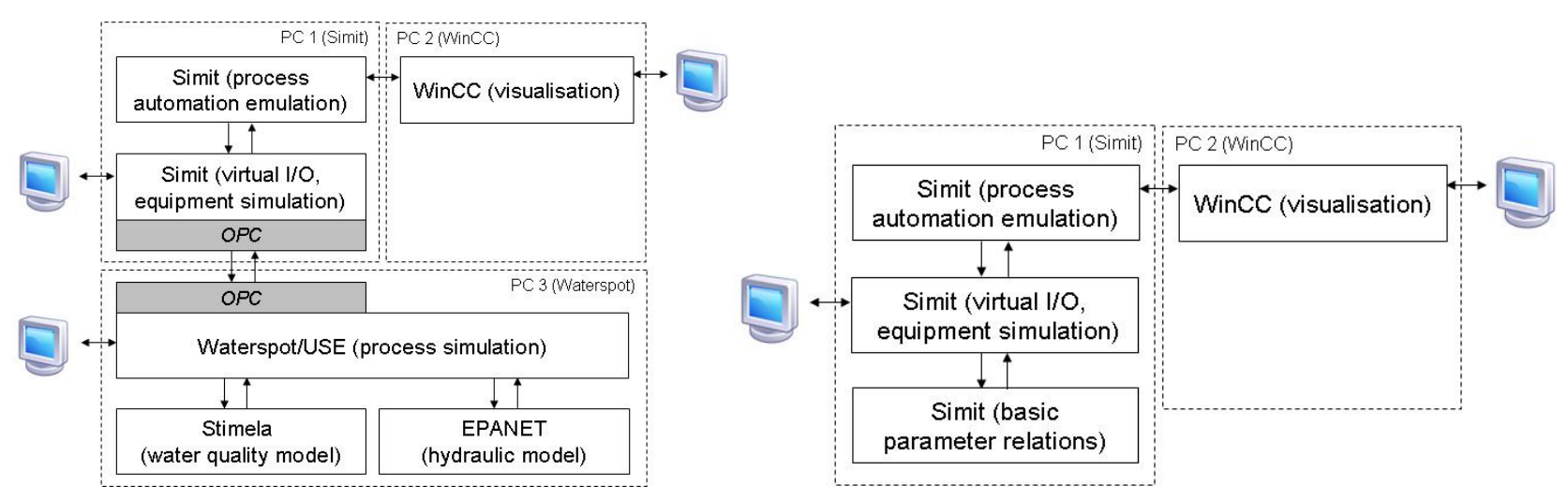

Figure 1. Setup of the AVC test system (left) and the VC test system (right).

As shown in Figure 1, the setup of the VC-system equaled the AVC-system, but instead of the process simulation models, a programmer defined twenty basic parameter relations within Simit, e.g. the relation between a pump's speed and the flow. For several parameters, virtual sliders were set up in Simit, as shown in Figure 2. 


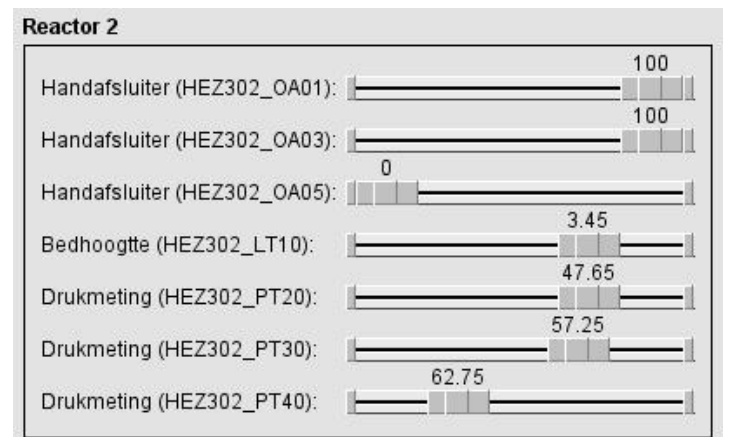

Figure 2. Virtual sliders for sensors and actuators of Reactor 2 in the VC system (labels in Dutch, 'Handafsluiter' means manually operated valve, 'Bedhoogte' means bed height, and 'Drukmeting' means pressure measurement).

\section{Experimental set-up}

For the AVC-system, two hydraulic model environments were available; EPANET, part of the Waterspot simulator and Flownet, a Simit plug-in. EPANET and Flownet were compared using a multi criteria evaluation. Each criterion exists of one or more sub criteria as Table 2 shows. Each sub criterion has the same weight. The sum of the scores of the sub criteria determines the score for the criterion. Each criterion has the same weight.

Table 2. Evaluation criteria EPANET versus Flownet

\begin{tabular}{ll}
\hline Criterion & Sub criteria \\
\hline Efforts to set up new model & $\begin{array}{l}\text { Setup of a pipe, setup of a weir, setup of a pump, } \\
\text { time to set up model, manual }\end{array}$ \\
Transparency and maintainability models & Help files, user interface, support, units \\
Efforts to integrate with Simit & Interface type \\
Robustness & Accuracy, robustness \\
Costs/licenses & Price, license restrictions \\
\hline
\end{tabular}

An optimized control strategy was designed for the existing control of the pellet softening treatment step of drinking water treatment plant Wim Mensink at Wijk aan Zee, owned and operated by PWN (Worm, under review). Prior to the experiment a programmer, who was not involved in the experiment, put 13 errors in the software. Two different test teams did the functional test of the software simultaneously, one team using the AVC-system, the other team using the VC-system. The composition of the Design team, Team AVC and Team VC is shown in Table 3. To limit the advantage of foreknowledge of the software, the software designer and the programmer who built the software were not in the same test team.

Table 3. Composition of the Design team and the test teams.

\begin{tabular}{lll}
\hline Design team & Team AVC & Team VC \\
\hline Programmer A & Programmer B & Programmer A \\
Software designer A & Software designer A & Software designer B \\
& Operation supervisor A & Operation supervisor B \\
\hline
\end{tabular}

The teams were asked to perform the functional test as they would have done in reality. Suggestions to the test teams during the experiment were minimized and test teams were able to determine their approach. A functional test protocol was available for the test teams during the experiment. The protocol was defined without knowledge of the place or type of errors put into the software. On request, the test protocol was extended for Team AVC to test and optimize the caustic soda dosage controller. The participants knew errors were put in the software, but not the exact number.

During the experiment each test item in the protocol was classified 'good', 'wrong' or 'not possible' by the operation supervisor. 'Not possible' could be selected when a test item could not be evaluated. For the experiment two evaluation-parameters were defined, i) the number of 
deliberately inserted errors found by the test teams and ii) the number of test items classified 'wrong' by the test teams. For the latter a Chi square test was executed with two samples and categories 'good' and 'wrong', yielding one degree of freedom. The null hypothesis was that the test results of the AVC team equaled the test results of the VC team and was preferably rejected, to be able to differ between the AVC test method and the VC test method. The test items classified 'not possible' were excluded from this evaluation, because they did not contribute to finding errors. Still, fewer 'not possible' items indicated a wider scope of the test system. After the experiment, each member of the test teams filled out a questionnaire containing propositions related to the contribution of the process simulation models to the results of the test on a five-point Likert scale (Likert, 1932) ranging from 1 (strongly disagree) to 5 (strongly agree). Three out of the ten propositions were for Team AVC exclusively.

\section{RESULTS}

When comparing EPANET and Flownet, EPANET scored higher on 'efforts to set up new model', 'transparency and maintainability models' and 'costs/licenses', Flownet scored higher on 'efforts to integrate with Simit'. EPANET and Flownet balanced on 'robustness'. EPANET was preferred over Flownet.

Table 4 shows the results of the test teams for the thirteen deliberately inserted errors. Four of these errors appeared to be put in unmodified parts of the software. During the functional test, team AVC identified one of these four errors, an unrealistic high maximum value of the total hardness measurement. The other three remained undiscovered. Team VC decided not to test unmodified software. Of the remaining nine errors, the Design team found eight during the unit test. Only team VC identified the ninth, an old tag code in an alarm presentation, during the functional test.

Table 4. Test results for the errors put into the software deliberately prior to the experiment.

\begin{tabular}{lllll}
\hline & Total & $\begin{array}{l}\text { Found by design team } \\
\text { during unit test }\end{array}$ & $\begin{array}{l}\text { Found by team AVC } \\
\text { during functional test }\end{array}$ & $\begin{array}{l}\text { Found by team VC } \\
\text { during functional test }\end{array}$ \\
\hline $\begin{array}{l}\text { Errors in unmodified } \\
\text { parts of the software }\end{array}$ & 4 & & 1 & 1 \\
$\begin{array}{l}\text { Errors in modified parts } \\
\text { of the software }\end{array}$ & 9 & 8 & & $\mathbf{1}$ \\
\hline Total & $\mathbf{1 3}$ & $\mathbf{8}$ & $\mathbf{1}$ & \\
\hline
\end{tabular}

Team AVC evaluated 144 test items, Team VC 83 items. This difference was caused by the extended test protocol of Team AVC and by the fact that Team AVC tested unmodified software as well. During the functional test, 78 items were tested by both Team AVC and Team VC. The results of these 78 tests are shown in 
Table 5. The test item on the continuation of the RO-water dosing after switching of a reactor when filling of the tank was interrupted, was tested 'wrong' by both Team AVC and Team VC. The other 'wrong' items were mentioned by a single team. The null hypothesis of the Chi square test could be rejected, meaning the test results of team AVC differ from the test results of team VC with significance $p=0.21$. The AVC team judged $1 \%$ of the test items 'not possible', the VC team 17\%, indicating a scope limitation of the VC system, compared to the AVC system. Most of the "not possible' test items of Team VC contained process consequences of actions, which were not simulated with the basic parameter relations, e.g. the height of the fluidized bed decreased when the flow through the reactor decreased. 
Table 5. Judgement of the items tested by both team AVC and VC during the functional tests.

\begin{tabular}{|c|c|c|c|c|c|c|}
\hline \multirow[t]{2}{*}{ Test } & \multicolumn{3}{|c|}{ Team AVC } & \multicolumn{3}{|c|}{ Team VC } \\
\hline & Good & Wrong & $\begin{array}{l}\text { Not } \\
\text { possible }\end{array}$ & Good & Wrong & $\begin{array}{l}\text { Not } \\
\text { possible }\end{array}$ \\
\hline Log in & 1 & & & 1 & & \\
\hline $\begin{array}{l}\text { Flushing with } R O \text {-water after } \\
\text { switching off a reactor }\end{array}$ & 31 & 3 & & 31 & 3 & \\
\hline Grain dosing & 12 & & & 10 & & 2 \\
\hline Pellet discharge & 20 & & & 9 & & 11 \\
\hline Control of flow through reactors & 6 & & & 6 & & \\
\hline Control of caustic soda dosage & 2 & & & 1 & 1 & \\
\hline Water on floor & 2 & & 1 & 3 & & \\
\hline Total & 74 & 3 & 1 & 61 & 4 & 13 \\
\hline
\end{tabular}

In the questionnaire five propositions were judged 'agree' or 'strongly agree' by four or five of the six participants, see Table 6 . These five propositions dealt with the added value of the Simit emulation platform and the added value of process behavior to the test system. Five out of the six participants were neutral or disagreed with the proposition that in ten years more process simulation models of treatment steps will be set up and connected to Simit. In Team AVC, two participants were 'neutral' and one 'strongly agreed' with the statement that they are positive about their test system, all three participants of Team VC 'agreed'.

Table 6. Results questionnaire, total score (score Team AVC / score Team VC). 'Strongly disagree' was not chosen.

\begin{tabular}{|c|c|c|c|c|}
\hline & $\begin{array}{l}\text { Strongly } \\
\text { agree }\end{array}$ & Agree & Neutral & $\begin{array}{l}\text { Dis- } \\
\text { agree }\end{array}$ \\
\hline I prefer Simit over PLCSIM ${ }^{1}$ for software testing & $4(2 / 2)$ & $1(1 / 0)$ & $1(0 / 1)$ & \\
\hline I prefer Simit over hardware PLCs for software testing & $1(1 / 0)$ & $4(1 / 3)$ & & $1(1 / 0)$ \\
\hline The addition of process behavior to the test system is valuable ${ }^{1}$ & $1(1 / 0)$ & $5(2 / 3)$ & & \\
\hline The addition of process behavior accelerates software testing & & $4(1 / 3)$ & $1(1 / 0)$ & $1(1 / 0)$ \\
\hline $\begin{array}{l}\text { The addition of process behavior to the test system yields to } \\
\text { higher software quality }\end{array}$ & $1(1 / 0)$ & $4(1 / 3)$ & & $1(1 / 0)$ \\
\hline $\begin{array}{l}\text { In ten years time we will have more process simulation } \\
\text { models connected to Simit }\end{array}$ & & $1(1 / 0)$ & $3(2 / 1)$ & $2(0 / 2)$ \\
\hline Mainly the hydraulic model contributes to the software test ${ }^{3}$ & & 1 & 1 & 1 \\
\hline $\begin{array}{l}\text { Mainly the water quality model contributes to the software } \\
\text { test }^{3}\end{array}$ & & & 2 & 1 \\
\hline The process simulation did not hinder the software test ${ }^{3}$ & 1 & 1 & & 1 \\
\hline My general opinion on testing with this system is positive & $1(1 / 0)$ & $3(0 / 3)$ & $2(2 / 0)$ & \\
\hline
\end{tabular}

\section{DISCUSSION}

Participants mentioned that the addition of basic parameter relations was mainly valuable to accelerate the test and thus increase the efficiency. The setup and validation of process simulation models requires significant efforts. For small and medium size enterprises these efforts might make virtual commissioning unattractive (Hoffmann et al., 2010), especially when working with the modeling platform requires substantial skills and knowledge. Process simulation models which describe only a limited part of a treatment plant, which are robust, easy to run, and easy to integrate with (emulated) PA-systems will not only boost the implementation of AVC-systems, but increase the life time of process simulation models (Hass et al., 2005) at the same time.

When looking at the errors put into the software, it became clear that the majority of the errors were revealed during the unit test, rather than during the functional test. This either indicates that the 
programmer who set up the errors lacked specific process knowledge, or that the majority of the errors was related to 'status I/O' or signal connections which did not influence the process directly. When focusing at the 'process $\mathrm{I} / \mathrm{O}$ ', observations during the experiment showed that not the exact values were relevant for the functional test, but the response to the passing of process thresholds. This can be understood from the fact that most drinking water treatment processes are relatively slow, meaning that a direct (within minutes) response is not necessary. This is different in drinking water distribution where a direct response to changes in pressure in the network is relevant. In general, the integration of process simulation models for AVC is most feasible when the highest risks can be limited.

The larger amount of 'not possible' test items for the Team VC, showed that with the AVC system more items could be evaluated. In that perspective, the addition of process behavior was valuable. But, the extra items tested by team AVC were mainly related to the performance of the process simulation, rather than the performance of the PA-software. Probably, the 'wrong' items, which were identified by the test teams, would have been found if no process behavior would have been present. Observations during and after the experiment showed that the personal preferences in the approach of the operation supervisor during a PA-software test influenced the results of the test noticeably. This assumption is supported by the fact that in the recent test of the software of the Heemskerk I membrane filtration plant, errors that were in the software for ten years were found by a different test team. The same casus supports the indication that the majority of software errors do not influence the process directly or noticeably.

\section{Recommendation}

In future, manufacturers of manufacturing systems will offer simulation models with new equipment (Hoffmann, 2010). In drinking water treatment this should be requested from suppliers of advanced treatment technologies like membrane filtration and advanced oxidation technologies, processes where a direct response to deviations is relevant, to set up AVC-systems.

\section{CONCLUSIONS}

In this paper the contribution of process simulation models to the FAT of PA-software has been studied, thus introducing advanced virtual commissioning of PA-software in drinking water treatment. The hypothesis was that with AVC more errors would be found during the FAT than with VC. The AVC-system, an integration of the Waterspot process simulator and emulated PAsoftware on the Simit platform was set up successfully and 93 process parameters were exchanged dynamically. The VC-system was set up being the Simit platform and basic parameter relations. Each test system was used by a different test team to evaluate the same piece of PA-software in a FAT. During the functional test, each test team found one (different) error of the thirteen errors put into the software deliberately. The majority of these errors was found by the Design Team during the unit test, because the majority of the $\mathrm{I} / \mathrm{O}$ signals are 'status I/O', and not 'process I/O'. The AVC-team using the system with process simulation found three errors, the VC-team using the system with basic parameter relations found four. The significance $p$ of the difference between the number of 'good' and 'wrong' tested items of both teams is 0.21 , more than the commonly used 0.05. It was concluded that the hypothesis that with AVC more errors could be found than with VC could not be accepted. And, that for the FAT of PA-software of drinking water treatment plants, the addition of basic parameter relations to PA-emulation satisfied. Not the exact process behavior helped to find errors, but the passing of process thresholds. Still, the AVC-team judged $1 \%$ of the test items 'not possible', the VC-team 17\%, indicating a wider test scope of the AVC-system, compared to the VC-system. 


\section{ACKNOWLEDGEMENTS}

Authors wish to thank Robert Nugteren for setting up the VC and AVC test environments, René Kerkvliet, Jeroen Vreman, Erwin Blokland, Rob van der Hulst and Gerard Admiraal for carrying out the experiment and Matthijs van der Wielen for his support.

\section{REFERENCES}

Bradu, B., Gayet, P., Niculescu, S.-I. (2009). A process and control simulator for large scale cryogenic plants. Contr. Eng. Pract., 17(12), 1388-1397.

Drath, R., Weber, P., Mauser, N. (2008). An evolutionary approach for the industrial introduction of virtual commissioning. IEEE Symposium on Emerging Technologies and Factory Automation, Hamburg, Germany.

Franke, R., Doppelhamer, J. (2007). Integration of advanced model based control with industrial IT. Hass, V. C., Kuhnen, F., Schoop, K. M., Luis Puigjaner and Antonio, E. (2005). An environment for the development of operator training systems (OTS) from chemical engineering models. In: Computer Aided Chemical Engineering (ed.), Elsevier, 289-293.

Hoffmann, P., Schumann, R., Maksoud, T. M. A., Premier, G. C. (2010). Virtual commissioning of manufacturing systems a review and new approaches for simplification. 24th European Conference on Modelling and Simulation, Kuala Lumpur.

Krause, H. (2007). Virtual commissioning of a large LNG plant with the DCS „800XA“ by ABB. Eurosim, Ljubljana, Slovenia.

Likert, R. (1932). A technique for the measurement of attitudes. 22(140), 1-55.

Lucas, I. (2003). Testing times in computer validation. J. of Validat. Techn, 9(2), 153-161. Pellicciari, M., Andrisano, A., Leali, F., Vergnano, A. (2009). Engineering method for adaptive manufacturing systems design. Int. J. on Interact. Des. and Manuf., 3(2), 81-91.

Poon, P. L., Tse, T. H., Tang, S. F., Kuo, F. C. (2011). Contributions of tester experience and a checklist guideline to the identification of categories and choices for software testing. Softw. Qual. J., 19(1), 141-163.

Reinhart, G. and Wünsch, G. (2007). Economic application of virtual commissioning to mechatronic production systems. Prod. Eng. Res. Devel., 1(4), 371-379.

Rossman, L. A. (2000). EPANET 2 Users manual. Cincinnati, National Risk Management Research Laboratory, Water supply and water resources division.

Töbermann, J. C., Fischer, H. (2007). Virtuelle Inbetriebnahme mit Simit - Stand, Erfahrungen und Ausblick. 1980, 797-804.

Van der Helm, A. W. C., Rietveld, L. C. (2002). Modelling of drinking water treatment processes within the Stimela environment. Wat. Sci. Tech.: Wat. Sup., 2(1), 87-93.

Van Schagen, K. M. (2009). Model-based control of drinking-water treatment plants. thesis, Delft Centre for Systems and Control, Delft University of Technology.

Wildemann, H. (2005). Strategien der Digitalen Fabrik. Automobil Fachkongress 2005, Ludwigsburg, Germany.

Worm, G. I. M., Mesman, G. A. M., van Schagen, K. M., Borger, K. J., Rietveld, L. C. (2009). Hydraulic modelling of drinking water treatment plant operations. Drink. Water Eng. Sci., 2(1), 1520.

Worm, G. I. M., van der Helm, A. W. C., Lapikas, T., van Schagen, K. M., Rietveld, L. C. (2010). Integration of models, data management, interfaces and training support in a drinking water treatment plant simulator. Environ. Model. Softw., 25(5), 677-683.

Worm, G. I. M., Wuister, J. J. G., Van Schagen, K. M., Rietveld, L. C. (under review). Controldesign evaluation for drinking water treatment plants using a process model. J. of Wat. Sup.: Res. and Tech. - AQUA.

Zäh, M. F., Wünsch, G., Hensel, T., Lindworsky, A. (2006). Feldstudie - virtuelle inbetriebname. Zeitschrift fuer Wirtschaftlichen Fabrikbetrieb, 101(10), 595-599. 